\title{
A Ku-Band Low-Loss Traveling-Wave Power Divider using a Hollow Substrate Integrated Waveguide and Its Microstrip Transition
}

\author{
Sung-June Hong · Min-Pyo Lee · Seil Kim · Jun-Su Lim · Dong-Wook Kim*
}

\begin{abstract}
In this paper, we present a Ku-band low-loss traveling-wave power divider that uses a hollow substrate integrated waveguide (HSIW). For easy connection with microstrip-based devices and circuits, a low-loss transition between the microstrip line and the HSIW structure was implemented using $\mathrm{C}$-cut via holes at the discontinuity interface, which reduces radiation and leakage effects and improves mismatch performance. To validate the performance of the transition, a back-to-back microstrip-to-HSIW transition was designed, fabricated, and measured from $12.5 \mathrm{GHz}$ to $15.5 \mathrm{GHz}$. The measured results showed a return loss of $18 \mathrm{~dB}$ or more and an insertion loss of $0.5 \pm 0.07$ dB. An HSIW-based, low-loss 1:3 traveling-wave power divider was fabricated and measured from $13.5 \mathrm{GHz}$ to $14.5 \mathrm{GHz}$. The power divider showed a return loss of at least $21 \mathrm{~dB}$, an insertion loss of $0.57 \pm 0.03 \mathrm{~dB}$, and a power combining efficiency of $87.1 \%-88.3 \%$.
\end{abstract}

Key Words: Hollow Substrate Integrated Waveguide, Ku-Band, Power Divider, Transition, Traveling-Wave.

\section{INTRODUCTION}

A substrate integrated waveguide (SIW), which has a form similar to a rectangular waveguide, was proposed by Deslandes and $\mathrm{Wu}[1]$, who implemented it by placing via holes in parallel on a printed circuit board (PCB). The SIW suffers from a greater loss than a conventional rectangular waveguide because the substrate is filled with a dielectric material and has dielectric loss, but it has the advantage of easy integration with planar active and passive devices. To compensate for the dielectric loss of SIW, a hollow substrate integrated waveguide (HSIW), which removes the dielectric material and uses air as the transmission medium, was proposed [2]. In several studies, low-loss HSIWs have been analyzed for application to directional cou- plers, filters, and antennas [3-5].

A power divider typically has two kinds of configurations: a corporate structure and a traveling-wave structure [6]. The number of ports into which a corporate power divider can split the input power is $2^{\mathrm{n}}$ because the power divider uses binary power dividers, such as the Wilkinson power divider, branch line power divider, Lange power divider, and rat race power divider. By contrast, a traveling-wave power divider can split the power into an arbitrary number of ports, thereby facilitating its usage.

In this paper, we propose a low-loss microstrip-to-HSIW transition using $\mathrm{C}$-cut via holes and present a low-loss $\mathrm{Ku}$-band 1:3 traveling-wave power divider, which is based on an HSIW structure and uses the proposed transitions. The microwave performance of the transition and the power divider is validated

Manuscript received August 2, 2019 ; Revised November 12, 2019 ; Accepted March 5, 2020. (ID No. 20190802-061J)

Department of Radio and Information Communications Engineering, Chungnam National University, Daejeon, Korea.

"Corresponding Author: Dong-Wook Kim (e-mail: dwkim21c@cnu.ac.kr)

This is an Open-Access article distributed under the terms of the Creative Commons Attribution Non-Commercial License (http://creativecommons.org/licenses/by-nc/4.0) which permits unrestricted non-commercial use, distribution, and reproduction in any medium, provided the original work is properly cited.

(c) Copyright The Korean Institute of Electromagnetic Engineering and Science. All Rights Reserved. 
through electromagnetic simulations and measurements.

\section{MICROSTRIP-TO-HSIW TRANSITION}

Active and passive planar devices are generally microstriptype elements. A microstrip-to-HSIW transition is an essential component to reduce discontinuity at the interface of two different transmission structures. Fig. 1 shows the microstrip-toHSIW transition proposed in this work. The proposed transition uses $\mathrm{C}$-cut via holes that are symmetrically placed at the interface of the microstrip line and HSIW, thus reducing radiation and leakage effects through the shielding of via holes, and improving impedance matching. The $\mathrm{C}$-cut via holes can be replaced with triangular, rectangular, or other geometric shapes, as the specific shape is not critical for microwave performance.

In Fig. $1, w_{m s}$ is the width of a $50 \Omega$ microstrip line, $d$ is the diameter of a via hole, and $p$ is the distance between adjacent via holes. If $d$ and $p$ satisfy the conditions of (1) and (2), and the metallic loss is neglected, the radiation loss of two parallel via holes is less than $0.008 \mathrm{~dB}$ per wavelength. Therefore, the $d$ and $p$ values and the parameters of the $\mathrm{C}$-cut via holes should be determined using (1) and (2) [7]. $w_{t, m s}, l_{t, m s}, w_{t}$, $l_{t}$, and $w_{t i}$ are the design parameters, and their initial values are estimated from (3) to (7) [8]. $l_{t, m s}$ and $l_{t}$ determine the center frequency of the transition, and $w_{t, m s}, w_{t}$, and $w_{t i}$ are related to the impedance matching and bandwidth. $\lambda_{g 0}$ and $\lambda_{g, m s}$ are the wavelength in free space and the guided wavelength of the microstrip line, respectively. The initial values of all the design parameters were calculated from the design equations and then optimized using electromagnetic simulations. Iterative optimization results in optimal design parameters for the bandwidth and return loss.

$$
\begin{gathered}
d<0.2 \lambda_{g} \\
d / p \geq 0.5 \\
w_{t, m s}=4 w_{t r} \\
l_{t, m s}=\frac{\lambda_{g, m s}}{4} \\
w_{t}=1.2 w_{t, m s}
\end{gathered}
$$

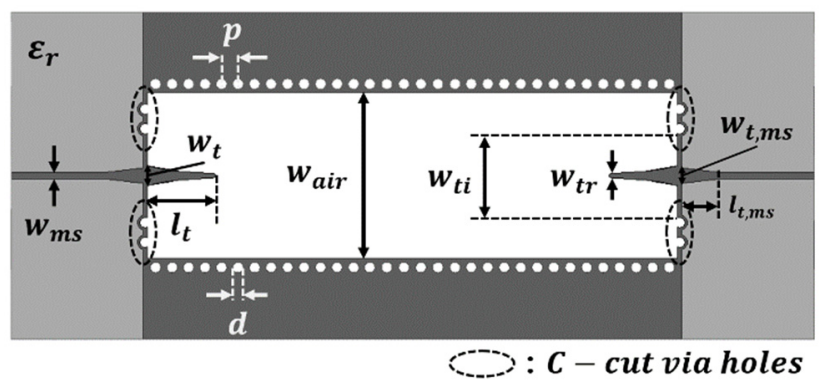

Fig. 1. Microstrip-to-HSIW transition with $\mathrm{C}$-cut via holes.

$$
\begin{gathered}
l_{t}=\frac{\lambda_{g 0}}{4} \\
w_{t i}=\frac{w_{a i r}+w_{t}}{2}
\end{gathered}
$$

The microstrip-to-HSIW transition was designed using a 12 mil-thick RO4003C substrate provided by Rogers Corporation, Chandler, AZ, USA. The design goals are listed in Table 1, and the optimized geometrical design parameters are shown in Table 2. The bandwidth of the transition was intentionally set to 2 $\mathrm{GHz}$, which is wider than that of the power divider for satellite communication systems, and an insertion loss of $0.6 \mathrm{~dB}$ was required for the power combining efficiency of $85 \%$.

Fig. 2 is a photograph of the fabricated back-to-back microstrip-to-HSIW transition that occupies $55.5 \mathrm{~mm} \times 30 \mathrm{~mm}$ (based on the reference planes). The top and bottom metal planes of the transition were implemented using top and bottom jig plates for convenience, although they could be fabricated using a conventional multi-layer PCB process.

Fig. 3 compares the simulated and measured $S$-parameter results of the back-to-back microstrip-to-HSIW transition. The back-to-back transition had a return loss of $18 \mathrm{~dB}$ or more and an insertion loss of $0.5 \pm 0.07 \mathrm{~dB}$ from $12.5 \mathrm{GHz}$ to $15.5 \mathrm{GHz}$. The measured results were in good agreement with the simulated results, although the resonance notches of $S_{11}$ slightly shifted to the lower frequency region by about $0.5 \mathrm{GHz}$.

Table 1. Design goals of the microstrip-to-HSIW transition

\begin{tabular}{lc}
\hline & Value \\
\hline Frequency $(\mathrm{GHz})$ & $12.5-15.5$ \\
Return loss $(\mathrm{dB})$ & $\geq 20$ \\
Insertion loss $(\mathrm{dB})$ & $\leq 0.6$ (back-to-back) \\
\hline
\end{tabular}

Table 2. Optimized geometrical design parameters of the microstrip-to-HSIW transition

\begin{tabular}{lc}
\hline Parameter & Value $(\mathrm{mm})$ \\
\hline$w_{m s}$ & 0.65 \\
$w_{t, m s}$ & 1.2 \\
$l_{t, m s}$ & 3.4 \\
$w_{t}$ & 1.8 \\
$l_{t}$ & 5.3 \\
$d$ & 1.0 \\
$p$ & 1.5 \\
$w_{a i r}$ & 15.2 \\
$w_{t i}$ & 7.6 \\
$w_{t r}$ & 0.4 \\
\hline
\end{tabular}




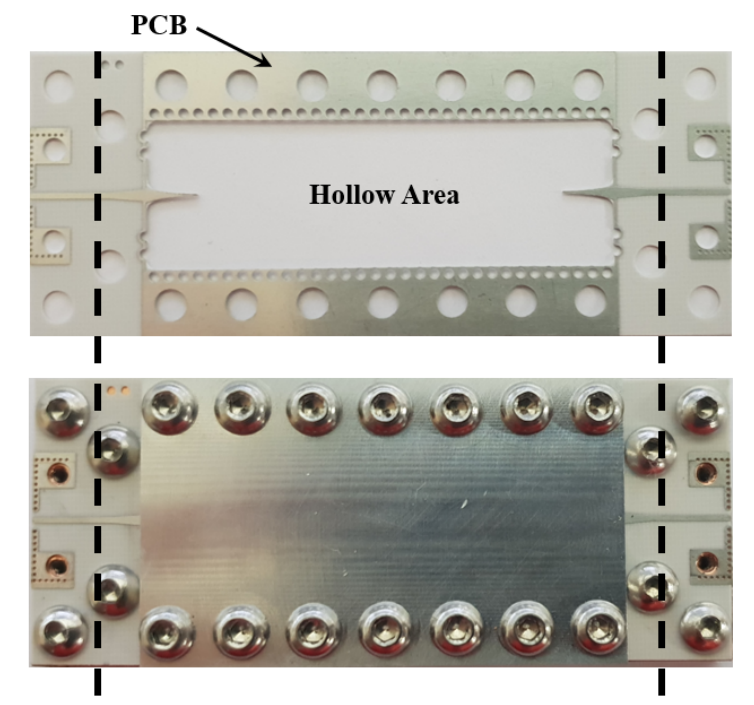

Reference Plane

Reference Plane

Fig. 2. Fabricated back-to-back microstrip-to-HSIW transition (upper: PCB part of the HSIW, lower: HSIW covered with aluminum jig plates).

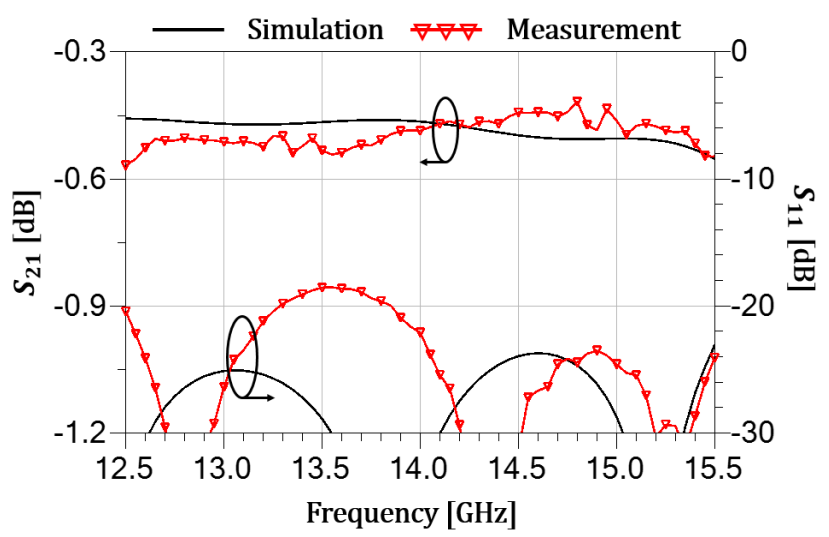

Fig. 3. Simulated and measured $S$-parameter results of the back-toback microstrip-to-HSIW transition.

\section{LOW-LOSS TRAVELING-WAVE POWER DIVIDER USING HSIW AND MICROSTRIP-TO-HSIW TRANSITION}

Fig. 4 shows a T-junction power splitter, which is conventionally used in a traveling-wave power-dividing configuration. For the traveling-wave power divider, a $1: \mathrm{N}$ power divider requires (N-1) stages of the $\mathrm{T}$-junction power splitters. In the schematic $\mathrm{T}$-junction power splitter in Fig. 4, $\mathrm{N}$ is the total number of power-splitting ports, and $\mathrm{S}$ is the stage position number of the cascaded $\mathrm{T}$-junction power splitters [9]. The power-splitting ratio in each port is also indicated.

The design parameters of the $\mathrm{T}$-junction power splitter are shown in Fig. 5, where $\mathrm{w}$ is the width of the window, $p_{x}$ and $p_{y}$ are the post position parameters, and $r_{p}$ is the radius of the post inserted near the $\mathrm{T}$-junction. By tuning three main design parameters, namely $w, p_{x}$, and $p_{y}$, to determine an impedance

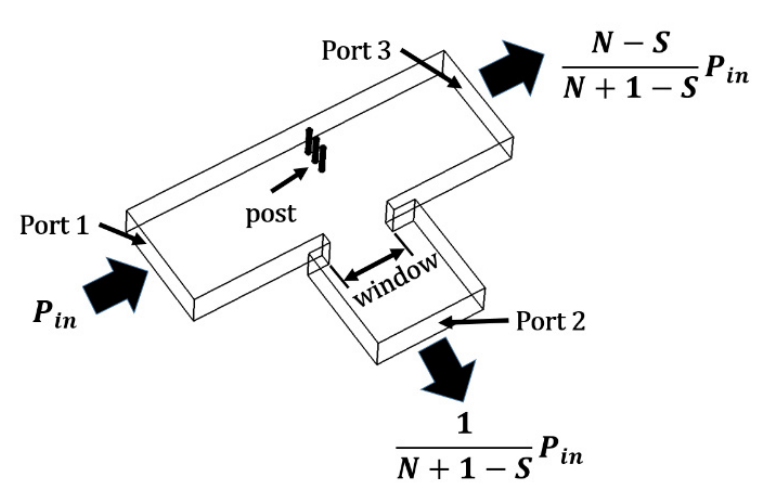

Fig. 4. T-junction power splitter in a traveling-wave power divider.

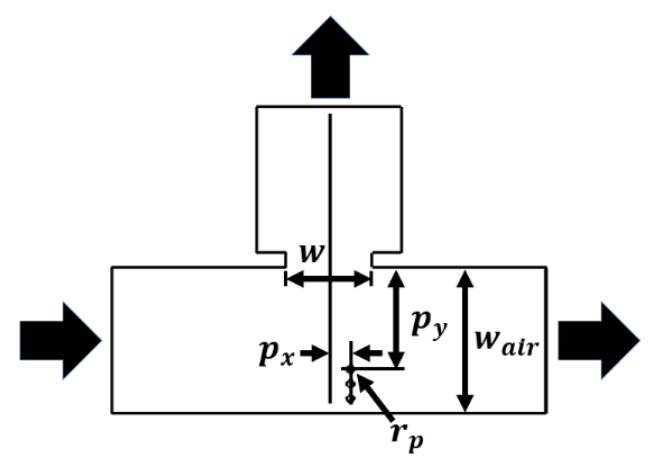

Fig. 5. Design parameters of a T-junction power splitter.

matching condition and the desired power-splitting ratio, the optimal parameter values for the design goals can be obtained from electromagnetic simulations.

The design and optimization procedures for each power splitter are described as follows:

(1) As the window width $w$ is the most important parameter in Fig. 5, it should be designed and optimized to determine the center frequency and the power-dividing ratio.

(2) After the window width $w$ is determined, the post position of $p_{x}$ is estimated and determined.

(3) If the parameters $w$ and $p_{x}$ are optimized and the approximate performance is obtained, the post position of $p_{y}$ is fine-tuned for optimal performance.

Fig. 6 shows two representative design parameters for the

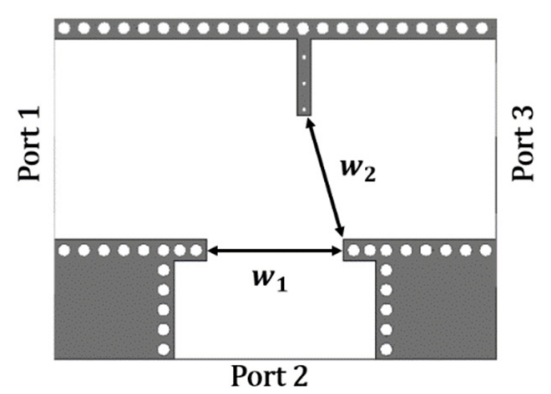

Fig. 6. Design parameters for the power-dividing ratio of the HSIW-based T-junction power splitter. 
power-dividing ratio of the HSIW-based T-junction power splitter. The geometrical parameter $w_{1}$ depends on the design center frequency, and the width ratio of $w_{1}$ and $w_{2}\left(w_{2} / w_{1}\right)$ mainly determines the power-dividing ratio of port 2 and port 3 . The width ratio can be expressed as (8), which is shown as a dotted line, and it is compared with the symbols with y error bars in Fig. 7. The error bar symbols were calculated from 3D electromagnetic simulations and were in good agreement with (8).

$$
w_{2} / w_{1}=0.36 \ln \left(2.235 \frac{S_{31}}{s_{21}}+0.52\right)+0.635
$$

Fig. 8 shows $w_{1}$ dependence on the frequency with varying power-dividing ratios of ports 2 and 3. $w_{1}$ decreases with the dividing ratio, ${ }_{31} / s_{21}$, and the center design frequency. The HSIW-based T-junction power splitter was designed by extracting the initial values from (8) and optimizing them using electromagnetic simulations.

Fig. 9 shows the schematic diagram of our designed 1:3 traveling-wave power divider, which is based on an HSIW structure. To prevent the operating frequency shift and the deviation of the power-dividing ratio due to the transition, the transition in each port was included in the stage design process, and three designed stages were cascaded to complete the $1: 3$ power divider. First, we set the required power-dividing ratio for each stage and found the design parameter values for it from (3) to (8) and the electromagnetic simulations. The first stage (stage 1) had a ratio of 1:2, and the second stage (stage 2) had a ratio of 1:1. The last stage (stage 3) used a $90^{\circ}$ curved bend, so that ports 2,3 , and 4 were on the same reference plane.

The Ku-band HSIW-based 1:3 traveling-wave power divider was designed using the $\mathrm{RO} 4003 \mathrm{C}$ substrate, on which the microstrip-to-HSIW transition had been implemented. The design goals are listed in Table 3 , and the optimized design para-

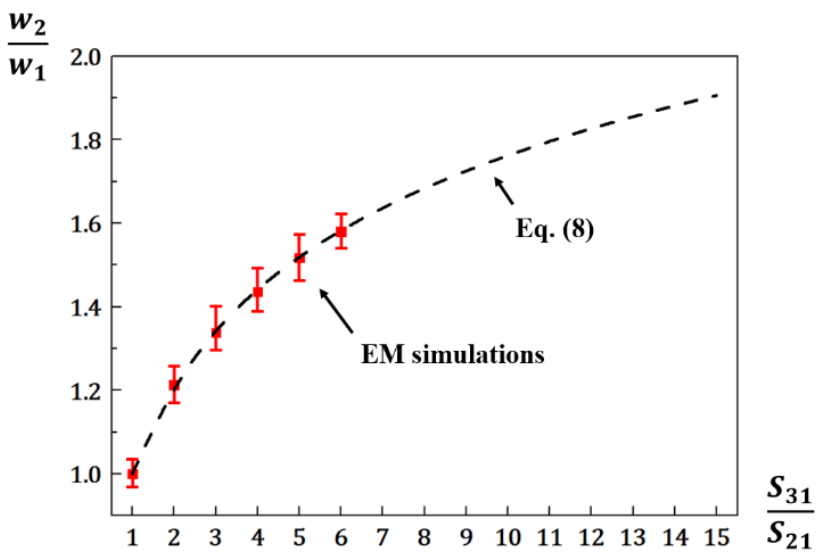

Fig. 7. Ratio of $w_{1}$ and $w_{2}\left(w_{2} / w_{1}\right)$ with the power-dividing ratio of ports 2 and $3\left(S_{31} / S_{21}\right)$. The dotted line indicates the values from Eq. (8), and the symbols with y error bars indicate the value ranges extracted from the electromagnetic simulations.

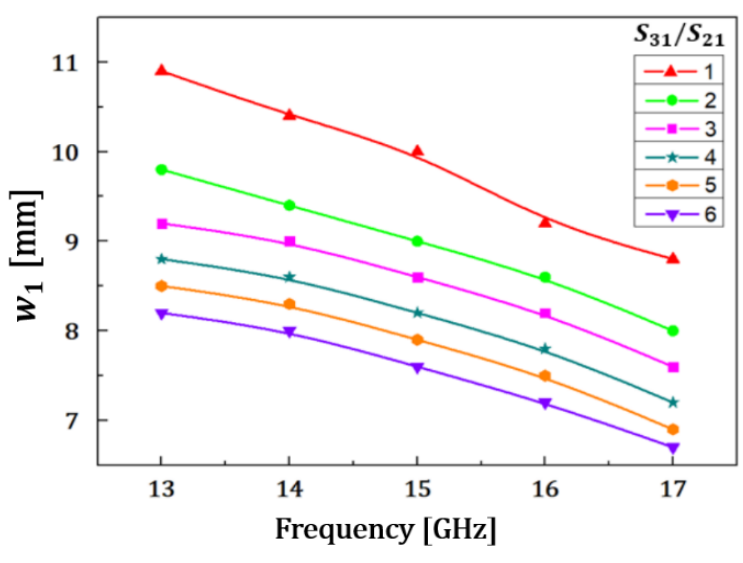

Fig. 8. Frequency dependence of the design parameter $w_{1}$ with the varying power-dividing ratios of ports 2 and 3 .

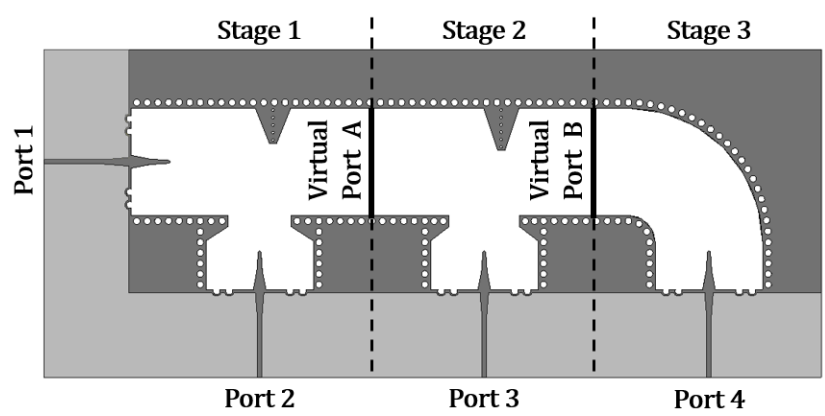

Fig. 9. A PCB layout of the HSIW-based 1:3 traveling-wave power divider.

meters obtained from the simulations are shown in Table 4. As the back-to-back transition showed the measured insertion loss of $0.5 \pm 0.07 \mathrm{~dB}$, and as an additional path loss could be expected in the power divider, the insertion loss of $0.7 \mathrm{~dB}$ was set as the target value (Table 3), which corresponds to the power combining efficiency of $85 \%$.

Fig. 10(a), (b), and (c) show the simulated $S$-parameter results

Table 3. Design goals of the HSIW-based 1:3 traveling-wave power divider

\begin{tabular}{lc}
\hline & Value \\
\hline Frequency $(\mathrm{GHz})$ & $13.5-14.5$ \\
Return loss $(\mathrm{dB})$ & $\geq 20$ \\
Insertion loss $(\mathrm{dB})$ & $\leq 0.7$ \\
Combining efficiency $(\%)$ & $\geq 85$
\end{tabular}

Table 4. Optimized geometrical design parameters of the HSIWbased 1:3 traveling-wave power divider

\begin{tabular}{ccccc}
\hline Stage & $w(\mathrm{~mm})$ & $p_{x}(\mathrm{~mm})$ & $p_{y}(\mathrm{~mm})$ & $r_{p}(\mathrm{~mm})$ \\
\hline 1 & 9.6 & 1.9 & 11.1 & 0.2 \\
2 & 10.6 & 2.4 & 10 & 0.2 \\
3 & 15.2 & \multicolumn{3}{r}{ Radius of curve $=11.4 \mathrm{~mm}$} \\
\hline
\end{tabular}




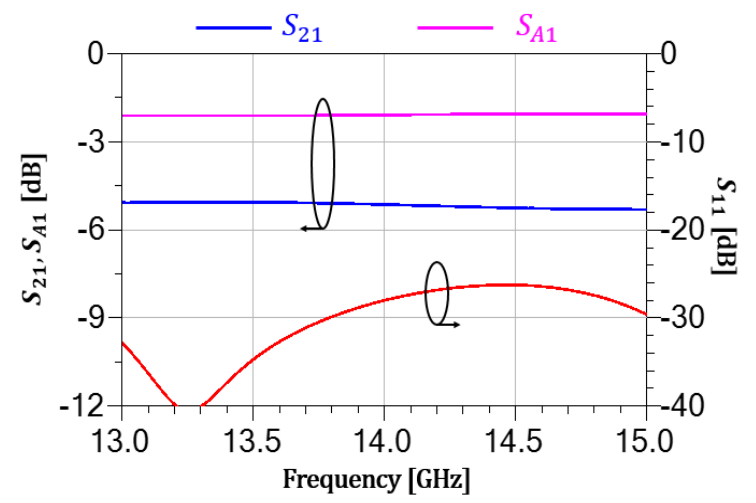

(a)

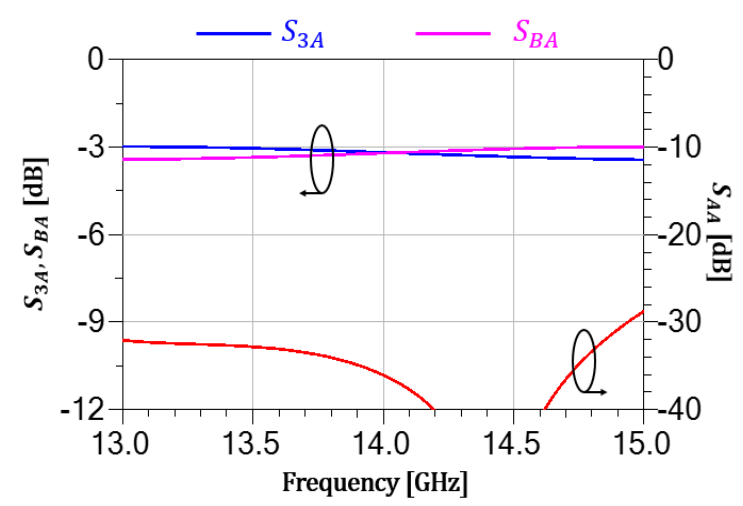

(b)

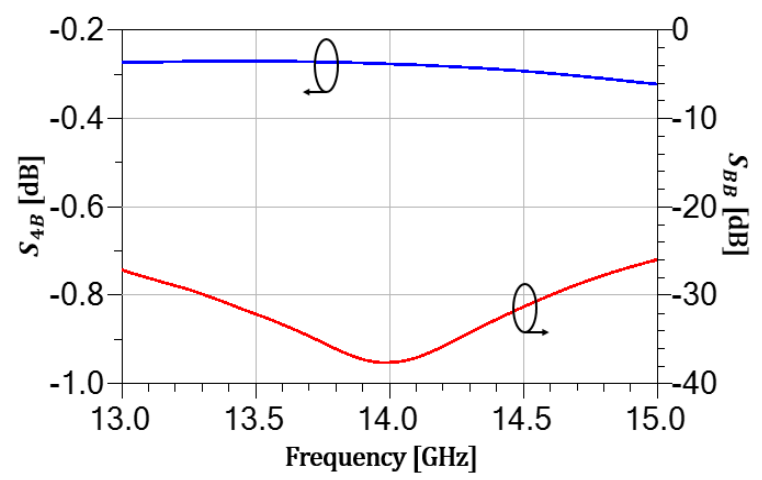

(c)

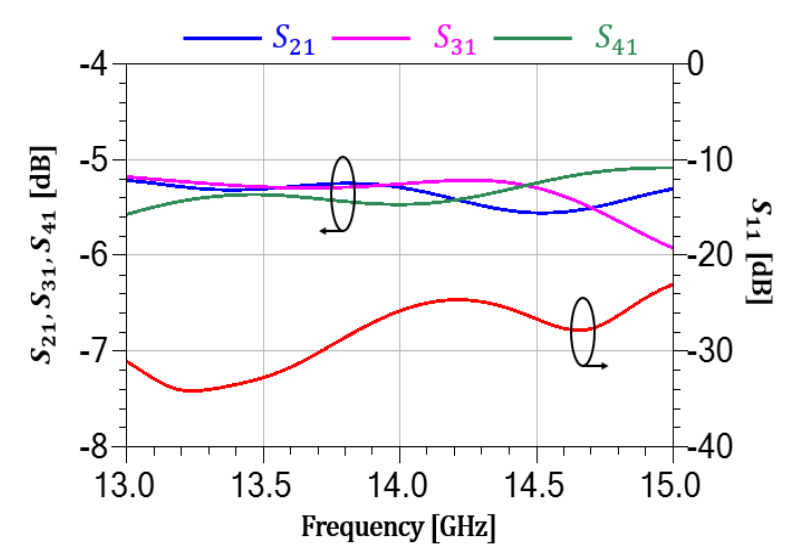

(d)

Fig. 10. Simulated $S$-parameter results of each stage and the complete HSIW-based 1:3 traveling-wave power divider. (a) Stage 1 , (b) stage 2, (c) stage 3, and (d) complete 1:3 traveling-wave power divider. for stages 1, 2, and 3, respectively. Fig. 10(d) shows the simulated results of the complete 1:3 power divider. Each designed stage had a return loss of $25 \mathrm{~dB}$ or more in the frequency range of 13.5-14.5 GHz and achieved the desired power-dividing ratios. The complete HSIW-based 1:3 power divider had a return loss of $24 \mathrm{~dB}$ or more and a transmission performance $\left(S_{21}, S_{31}\right.$, $S_{41}$ ) of $-5.4 \pm 0.15 \mathrm{~dB}$ from $13.5 \mathrm{GHz}$ to $14.5 \mathrm{GHz}$. The frequency response of the power divider was somewhat narrower than that of each stage because the frequency bandwidth of the cascaded stages was typically narrower than that of a single stage. In the frequency range of $13.5-14.5 \mathrm{GHz}$, it had an insertion loss of about $0.57 \mathrm{~dB}$ and a power combining efficiency of $87.2 \%$ or more, which met our design goals.

\section{FABRICATION AND MEASUREMENT}

Fig. 11 shows the fabricated 1:3 traveling-wave power divider, the signals of which are divided in the HSIW structure. Estimated from the reference plane, the size of the power divider is $101.3 \times 37.7 \mathrm{~mm}^{2}$, with the top and bottom metal planes formed using the jig itself after the PCB fabrication in the same manner as the microstrip-to-HSIW transition.

Fig. 12 compares the simulated and measured $S$-parameters of the HSIW-based 1:3 traveling-wave power divider. The measured results showed that the power divider had a return loss of $21 \mathrm{~dB}$ or more and a power-dividing ratio of $-5.3 \pm 0.3$ $\mathrm{dB}$ in the frequency range of $13.5-14.5 \mathrm{GHz}$. The range of the power-dividing ratio widened slightly because of the $\mathrm{PCB}$ process tolerance. Our simulation showed that the proposed power divider in this work was somewhat sensitive to the width of the window and the post positions in Fig. 5.

Fig. 13 compares the simulated and measured insertion loss
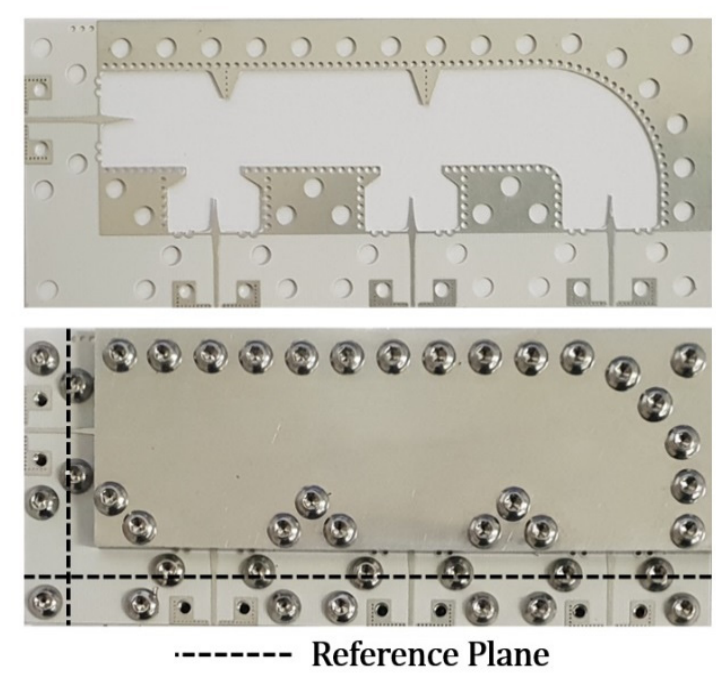

Fig. 11. Fabricated HSIW-based 1:3 traveling-wave power divider (upper: PCB part of the HSIW, lower: HSIW covered with aluminum jig plates). 


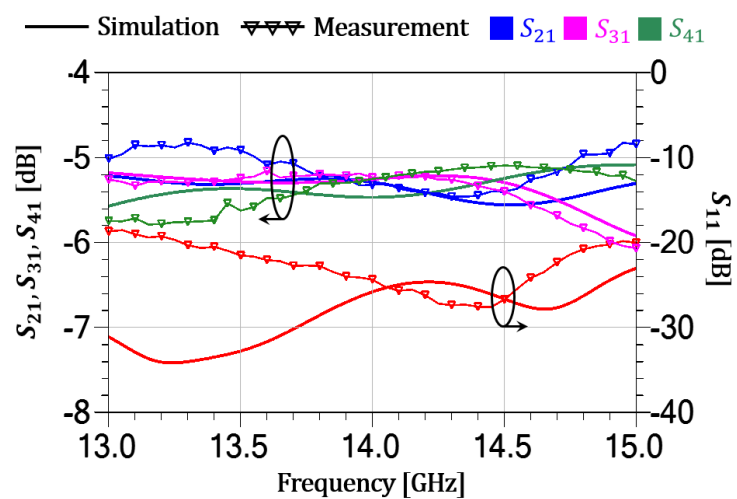

Fig. 12. Simulated and measured $S$-parameter results of the HSIW-based 1:3 traveling-wave power divider.

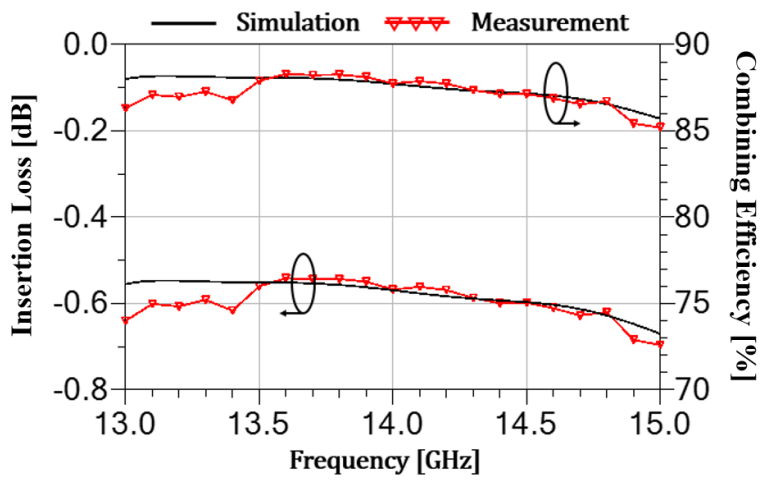

Fig. 13. Simulated and measured insertion loss and power combining efficiency of the HSIW-based 1:3 traveling-wave power divider.

and power combining efficiency of the HSIW-based 1:3 traveling-wave power divider. The power divider had a measured insertion loss of $0.57 \pm 0.03 \mathrm{~dB}$ and a measured power combining efficiency of $87.1 \%-88.3 \%$ from $13.5 \mathrm{GHz}$ to $14.5 \mathrm{GHz}$, consistent with the simulated results.

We compared our measured results with those of previously published power dividers in Table 5 [10,11]. Compared with the results in literature [10] that used waveguide-type components, the insertion loss of our 1:3 power divider increased by $0.2 \mathrm{~dB}$, and the power combining efficiency decreased by $3 \%$. As it used only one transition, [10] seemed to have a similar performance to our work if one transition loss was added, but it had a wider frequency performance. The comparison of our work with the corporate power divider in [11] also showed that our work improved the insertion loss by $1.4 \mathrm{~dB}$ and the power combining efficiency by $24 \%$.

Our HSIW-based power divider has the advantages of low cost and mass production due to the simple PCB process. Therefore, it is promising for applications requiring both high performance and competitive cost.

\section{CONCLUSIONS}

A microstrip-to-HSIW transition using C-cut via holes and
Table 5. Comparison of our study results with those of previously published power dividers

\begin{tabular}{lccc}
\hline & $\begin{array}{c}\text { Current } \\
\text { work }\end{array}$ & {$[10]$} & {$[11]$} \\
\hline Configuration & $\begin{array}{c}\text { Traveling- } \\
\text { wave type }\end{array}$ & $\begin{array}{c}\text { Traveling- } \\
\text { wave type }\end{array}$ & Corporate \\
Transmission & HSIW & Waveguide & SIW \\
Dividing ratio & $1: 3$ & $1: 4$ & $1: 4$ \\
Frequency $(\mathrm{GHz})$ & $13.5-14.5$ & $12.5-17.8$ & $9-10.5$ \\
Return loss (dB) & $\geq 21$ & $\geq 10$ & $\geq 17.5$ \\
Insertion loss $(\mathrm{dB})$ & $\leq 0.6$ & $\leq 0.42$ & $\leq 2$ \\
Combining & $87.1-88.3$ & $\geq 90.7$ & $\geq 63.1$ \\
$\quad$ efficiency $(\%)$ & & & \\
Transmission at & MS / MS & WG / MS & MS / MS \\
input/output port & $($ with two & (with one & (with two \\
& transitions) & transition) & transitions) \\
Substrate & RO4003C & DiClad 880 & Taconic \\
& & & RF-35 \\
$\varepsilon_{r}$ & 3.55 & 2.2 & 3.5 \\
tan $\delta$ & 0.0027 & 0.0009 & 0.0018 \\
Thickness (mm) & 0.305 & 0.254 & 0.254 \\
\hline
\end{tabular}

$\mathrm{MS}=$ microstrip, $\mathrm{WG}=$ waveguide.

an HSIW-based 1:3 traveling-wave power divider employing the proposed transitions were demonstrated for $\mathrm{Ku}$-band applications. The fabricated back-to-back transition showed a return loss of $18 \mathrm{~dB}$ or more and an insertion loss of $0.5 \mathrm{~dB}$ from 12.5 $\mathrm{GHz}$ to $15.5 \mathrm{GHz}$. The power divider using the transitions had a return loss of more than $21 \mathrm{~dB}$, an insertion loss of $0.57 \mathrm{~dB}$, and a power combining efficiency of $87.1 \%$ or more from 13.5 $\mathrm{GHz}$ to $14.5 \mathrm{GHz}$. The developed traveling-wave power divider using the HSIW structure can be effectively used in Ku-band applications that require low loss and small size in addition to high performance.

This work was supported by research fund of Chungnam National University.

\section{REFERENCES}

[1] D. Deslandes and K. Wu, "Integrated microstrip and rectangular waveguide in planar form," IEEE Microwave and Wireless Components Letters, vol. 11, no. 2, pp. 68-70, 2001.

[2] N. Ranjkesh and M. Shahabadi, "Reduction of dielectric losses in substrate integrated waveguide," Electronics Letters, vol. 42, no. 21, pp. 1230-1232, 2006.

[3] M. D. Fernandez, J. A. Ballesteros, and A. Belenguer, "De- 
sign of a hybrid directional coupler in empty substrate integrated waveguide (ESIW) ," IEEE Microwave and Wireless Components Letters, vol. 25, no. 12, pp. 796-798, 2015.

[4] R. M. A. Lee, J. Jin, I. Robertson, and A. Farzamnia, "Integrated waveguide slot antenna and cavity filter using hollow SIW in LTCC technology," in Proceedings of 2015 1st International Conference on Telematics and Future Generation Networks (TAFGEN), Kuala Lumpur, Malaysia, 2015, pp. 82-85.

[5] C. G. Hynes and R. G. Vaughan, "Empty substrate integrated waveguide antenna array," in Proceedings of 2018 IEEE International Symposium on Antennas and Propagation छ USNC/URSI National Radio Science Meeting, Boston, MA, 2018, pp. 1027-1028.

[6] K. J. Russell, "Microwave power combining techniques," IEEE Transactions on Microwave Theory and Techniques, vol. 27, no. 5, pp. 472-478, 1979.

[7] D. Deslandes and W. Wu, "Single-substrate integration technique of planar circuits and waveguide filters," IEEE

Sung-June Hong

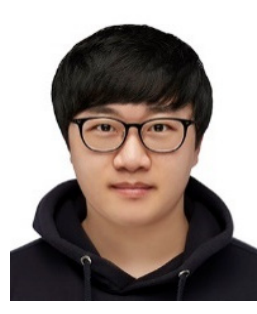

received his B.S. and M.S. degrees in Radio Science and Engineering from Chungnam National University, Daejeon, South Korea, in 2018 and 2020, respectively. He is currently working with SEMES Co. Ltd. His research interests include $3 \mathrm{D}$ printing technologies, hollow substrate integrated waveguides and their applications to microwave power amplifier modules.

Min-Pyo Lee

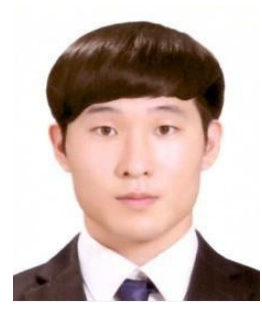

received his B.S. and M.S. degrees in Radio Science and Engineering from Chungnam National University, Daejeon, South Korea, in 2018 and 2020, respectively. He is currently working with i3system, Inc. His research interests include GaN HEMT wideband power amplifiers and their applications to jammer systems.
Transactions on Microwave Theory and Techniques, vol. 51, no. 2, pp. 593-596, 2003.

[8] H. Esteban, A. Belenguer, J. R. Sanchez, C. Bachiller, and V. E. Boria, "Improved low reflection transition from microstrip line to empty substrate-integrated waveguide," IEEE Microwave and Wireless Components Letters, vol. 27, no. 8, pp. 685-687, 2017.

[9] X. Jiang, "Two new Ka-band traveling wave power divider/combiner designs," M.S. thesis, North Carolina State University, Raleigh, NC, 2001.

[10] Y. Zhang, K. Yang, X. Ren, S. Xie, and L. Yimeng, "A study on novel broadband $\mathrm{Ku}$-band spatial power divider/combiner," in Proceedings of 2013 IEEE International Conference on Microwaves, Communications, Antennas and Electronic Systems (COMCAS), Tel Aviv, Israel, 2013, pp. 1-5.

[11] K. Song, W. Lu, S. Guo, and Y. Fan, "Four-way hybrid SIW/microstrip-line power divider with improved output isolation," Electronics Letters, vol. 55, no. 1, pp. 36-38, 2019.

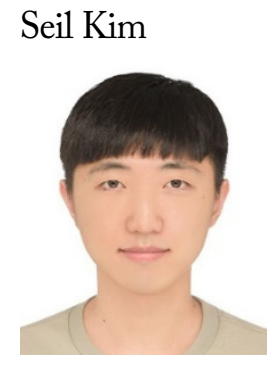

received his B.S. and M.S. degrees in Radio Science and Engineering from Chungnam National University, Daejeon, South Korea, in 2017 and 2019, respectively. $\mathrm{He}$ is currently working with Defense Agency for Technology and Quality. His research interests include GaN HEMT high power amplifiers and their applications to military radar modules.

Jun-Su Lim

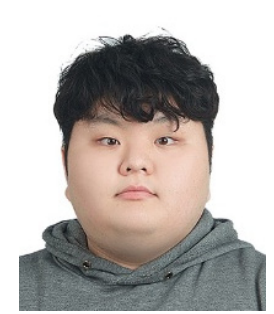

received his B.S. degree in Radio Science and Engineering from Chungnam National University, Daejeon, South Korea, in 2018. He is currently a M.S. student. His research interests include GaN HEMT microwave/millimeter-wave internally-matched power amplifiers and their applications. 


\section{Dong-Wook Kim}

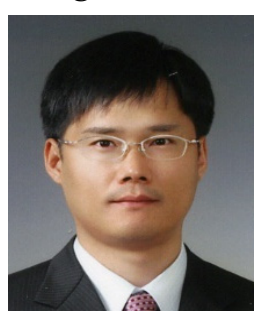

received his B.S. degree in electronic communications from Hanyang University, Seoul, South Korea, in 1990, and his M.S. and Ph. D degrees in electrical engineering from the Korea Advanced Institute of Science and Technology, Daejeon, South Korea, in 1992 and 1996, respectively. In 1996, he joined LG Electronics Research Center, Seoul, South Korea where he developed high-power III-V devices and monolithic microwave integrated circuits until 2000. From 2000 to 2002, he led R\&D teams to develop RF integrated passive devices on a thick oxidized Si substrate and their applications as a director of $\mathrm{R} \& \mathrm{D}$ center in Telephus, Inc. From 2002 to 2004, he was involved in the development of wireless security systems as a team leader in S1 Corporation, a company of the Samsung Group. In 2004, he joined the faculty of Chungnam National University, Daejeon, South Korea. In 2009, he was also with ETRI as an invited researcher. In 2010, he was a visiting scholar at the University of California at San Diego, La Jolla. He was the director of the Center for Information and Communication in Chungnam National University from 2016 to 2018, and is currently a full professor and also the director of Radiowave and Electrical Engineering Research Center. He has been an IEEE senior member since 2017. His research interests are GaAs- and GaN-based MMICs and internally matched power amplifiers, and microwave/millimeter-wave embedded modules, including miniaturized radar/sensor modules and ultra-wideband high-power modules. 